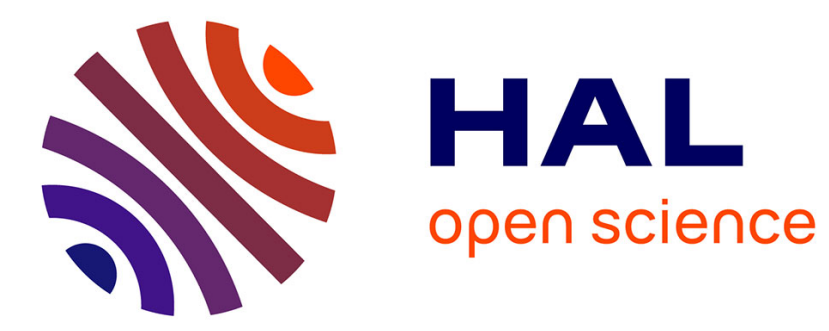

\title{
Dynamic structure factor near the glass transition: specific features
}

\author{
F. Mezei
}

\section{To cite this version:}

F. Mezei. Dynamic structure factor near the glass transition: specific features. Journal de Physique IV Proceedings, 1992, 02 (C2), pp.C2-31-C2-40. 10.1051/jp4:1992204 . jpa-00251275

\section{HAL Id: jpa-00251275 https://hal.science/jpa-00251275}

Submitted on 1 Jan 1992

HAL is a multi-disciplinary open access archive for the deposit and dissemination of scientific research documents, whether they are published or not. The documents may come from teaching and research institutions in France or abroad, or from public or private research centers.
L'archive ouverte pluridisciplinaire HAL, est destinée au dépôt et à la diffusion de documents scientifiques de niveau recherche, publiés ou non, émanant des établissements d'enseignement et de recherche français ou étrangers, des laboratoires publics ou privés. 


\title{
Dynamic structure factor near the glass transition: specific features
}

\author{
F. MEZEI
}

BENSC, Hahn-Meitner-Institut, Pf. 390128, 1000 Berlin 39, Germany

ABSTRACT

The dynamic structure factor in the supercooled liquid state just above the glass transition temperature reveals a number of characteristic universal features in the so-called "fragile" class of glassy systems. A theory independent, unbiased analysis shows that, compared to the behaviour expected in the ordinary liquid state, one can observe: (a) a two-step pattern in the time relaxation of the liquid structure factor, the slower of these steps scaling with the bulk viscosity, (b) a relatively small, oscillatory wavenumber dependence of the relaxation dynamics, and (c) approximate master functions of unconventional shapes for both relaxation steps. These qualitative features are in agreement with predictions of recent mode coupling theories and, in addition, the quantitative behaviour is also compatible with the theoretically predicted scaling properties.

\section{Introduction}

Recent years have wittnessed a considerable, conjugated experimental and theoretical effort towards the understanding of the basic physics of the glass transition. The studies concentrated on the peculiar dynamics of atomic motions in glassformers around and primarily above the conventional glass transition temperature $T_{g}$ (where solidification in an everyday sense occurs and thermal hysteresis sets in at the same time) in the supercooled liquid state. The observed dynamics represents archetypical examples of slow atomic motions as compared to the characteristic atomic collision times of $10^{-12}-10^{-13} \mathrm{sec}$. Their exploration by neutron scattering, which gives the most direct information in space and time, has been made possible by the evolution of high resolution spectroscopic methods, such as Neutron Spin Echo (NSE), which makes the $10^{-11}-10^{-8}$ sec time domain accessible.

The available extended set of neutron scattering data ${ }^{1-6}$ on a large variety of samples of the so-called "fragile" glassformer cathegory 7 reveals a remarkable compatibility with predictions of recent mode coupling (MC) theories 8-9. "Compatibility" means that by an adequate choice of parameters it is possible to fit experimental results within error to the predicted scaling relations. Unfortunately, the number of fit parameters is considerable, and therefore reduces the significance of these fits, particularly in view of the inherently limited 
statistical accuracy of neutron scattering data. A particular problem is the lack of a clear signature of the predicted critical temperature $\mathrm{T}_{0}>\mathrm{T}_{\mathrm{g}}$. This enhances the ambiguity of the comparison of theory and experiment, although the absence of any real divergence at the presumed critical temperature is well explained by the presence of the activated, jump-diffusion type of motions ${ }^{11}$.

It is the aim of the present analysis to point out the unusual features in the experimental results, which cannot be understood by analogy to the ordinary liquids or amorphous solid states 12 . We shall first outline most basic ideas and predictions of the MC theories and give an overview of the experimental behaviour. It will be shown that these unusual qualitative features specific of the vicinity of the glass transition are well explained by the MC predictions. As a matter of fact, no other theoretical interpretation is available as of today.

\section{Mode Coupling Theory: Basic Ideas}

Conventionally glasses are regarded as very high viscosity liquids. The glass transition temperature is therefore phenomenologically defined as the temperature where the viscosity attains $10^{13}$ poise. This high value corresponds to solid-like behaviour under ordinary forces. The temperature dependence of the viscosity provides the most elementary information about the transition. Two extreme types of behaviour can be observed and correspondingly the categories of "strong" and "fragile" glasses were introduced by A. Angell 7 . The former is characterized by an essentially Arrhenius type of temperature dependence (for which vitreous silica is the best known example). Here a covalent bond network is already present in the liquid state (hence the appellation "strong") and there is no apparent deviation from thermally activated dynamics.

To the contrary, on the other extreme of "fragile" glasses there is a very strong deviation from the Arrhenius behaviour, characterized by the bending region at temperatures of $1.1-1.5 \mathrm{~T} g$ between two roughly straight Arrhenius like sections. Such a crossover between two thermally activated regimes suggests that there are substantial underlying structural and/or dynamical changes in these systems, which in practice include a variety of ionic, molecular and polymeric glasses. The dramatic acceleration of the increase of the viscosity with decreasing temperature upon approaching $T_{g}$, compared with the slowish high temperature variation, is the subject of many recent studies on the glass transition. The first fundamental success of modern generalized hydrodynamic theories, which have become known as mode coupling (MC) theories after the most widely and successfully used approach, is that they can provide an explanation for this crossover. This explanation implies a critical dynamic instability in a manner which is analogous to ordinary second order phase transitions. Thus, for example, the bending region in. Fig. 1 would correspond to a power law behaviour $\eta \propto\left(\mathrm{T}-\mathrm{T}_{0}\right)^{-\gamma}$ for $\mathrm{T}>\mathrm{T}_{0} \simeq 1.1 \mathrm{~T}_{\mathrm{g}}$. There is, however, no observable divergence at $\mathrm{T}_{0}$, rather a thermally activated variation sets in again with a much higher slope (increased activation energy). Thus, the crossover from low to high activation energy regions would reflect an underlying profound change of the microscopic dynamics, which can be regarded as a phase transition.

The basic idea of mode coupling theories can be sketched as follows ${ }^{9}$ : In the 
Mori-Zwanzig formulation the time dependent density-density correlation function $\Phi_{q}(t)$ can be obtained by solving the Laplace transformed equation of motion

$$
\Phi_{q}(z)=-\left(z-\frac{\Omega_{q}^{2}}{z+i v}\right)^{-1}
$$

where $\Omega_{q}$ is a characteristic microscopic frequency (atomic vibrations) and the constant $v$ represents the stochastic friction in the simplest approximation. This equation describes damped harmonic oscillators and corresponds to the dynamics of an idealized simple viscous liquid. The generalization of this model consists of the very obvious observation that in a real system the friction term will not be a real constant, but it will rather reflect the detailed properties of the system, i.e. it is a $q$ and Laplace frequency $(z)$ dependent complex number:

$$
v=v(q, z)
$$

Since this $q$ and $z$ dependence is determined by the interaction coupling and the actual state of the system [the latter one being uniquely described by the correlation function $\left.\Phi_{q}(z)\right]$, we have

$$
i v(q, z)=F_{q}\left(w, \Phi_{k}(z)\right)
$$

where $W$ is a set of coupling constants. One can examine various mode coupling approximations by replacing this unknown functional $F_{q}$ by the first terms in a

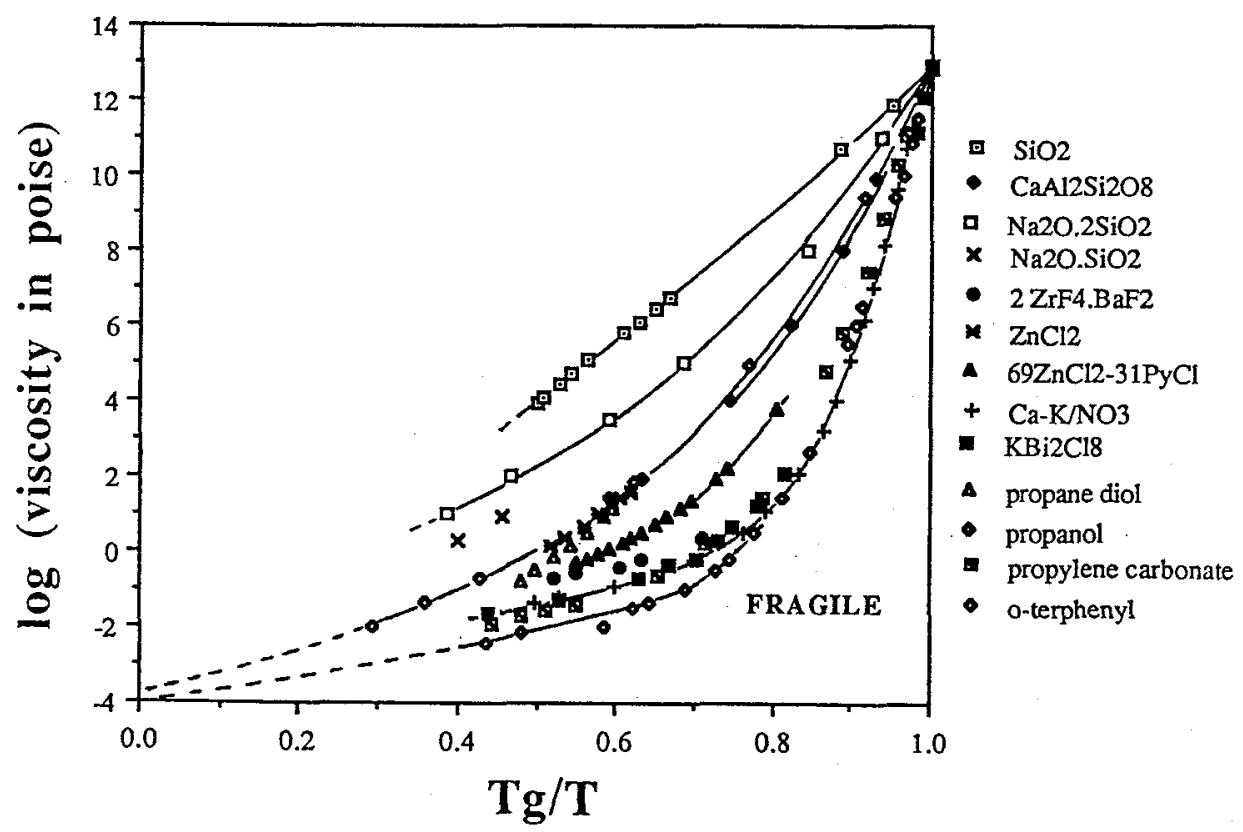

Fig. 1. Arrhenius plot of the temperature dependence of the viscosity with $T_{g}$ taken as temperature unit in various glasses ${ }^{7}$. The "strong" limit (straight line) corresponds to the Arrhenius behaviour, the "fragile" one to a strong bend between two relatively straight sections. 
Taylor expansion. For example, by also assuming, in order to simplify the mathematics, that $v$ would only depend on $\Phi_{k}$ at $k=q$, we can write

$$
i v(q, z)=i v+w_{1} \Phi_{q}(z)+w_{2} \Phi_{q}^{2}(z)+\ldots
$$

Thus, we get a non-linear equation for $\Phi_{q}(z)$, which can be solved analytically or numerically for various phenomenological coupling constant sets $w_{1}$, $w_{2}$, etc. It was discovered early on, that even such an oversimplified so-called "schematic" model can reproduce quite non-trivial features of the glass transition. Even the simplest model with only $w_{1} \neq 0$ is unusual $1^{\circ}$ : beyond a critical value of $w_{1}$ the correlation function $\Phi_{q}(t)$ will not relax to 0 at $t \rightarrow \infty$, as expected for a liquid in which structural correlations show only a finite lifetime, but $\Phi(t)$ tends to a finite value on $t \rightarrow \infty$, i.e. we obtain a frozen, glassy state which keeps its local structure for all times. ( $\Phi_{\mathrm{q}} \neq 0$ at $t \rightarrow \infty$ is the signature of non-ergodicity.) In the detailed analysis it turns out that the $w_{1} \neq 0, w_{2} \neq 0$ model is the simplest one, qualitatively comparable to the really observed glassy behaviour ${ }^{8}$. The important general point, however, is that three are critical values of the sets of $w_{n}$ (in general $w_{n}=w_{n}\left(q, k_{1} \ldots k_{m}\right)$ ) coupling constants at which the system becomes non-ergodic, i.e. frozen. Approaching adequate critical points (e.g. by changing external parameters, such as temperature) leads to power law divergences and scaling properties, analogously to ordinary critical phenomena. This, as indicated above, can explain the crossover in the temperature dependence of the viscosity in fragile class glasses. We cannot, however, expect fully developed divergences, since the criticality only applies to the continuous hydrodynamic motions and not to the atomic jump processes, which, in this framework, become responsible for the finite value and the evolution of the viscosity at $T \leqslant T_{0}$ and thus around $T_{g}$.

Rather than enumerating a list of predictions of the MC theories relevant to the experimental studies discussed here, we will invoke below these predictions directly at the discussion of the various experimental findings.

\section{Main unusual experimental features: the " $\beta$ " relaxation}

In what follows, we shall analyse neutron scattering results $1,2,6$ of $\mathrm{K}-\mathrm{Ca}-\mathrm{NO}_{3}(\mathrm{KCN})$, the first and best studied example, which proved to be a representative example of the variety of samples investigated by now.

One most remarkable experimental finding, as revealed by Time-of-Flight spectroscopy, is the anomalous increase of the apparent density of states at energies below $5 \mathrm{meV}$ starting at $\mathrm{T}_{\mathrm{g}} \simeq 60^{\circ} \mathrm{C}$ with increasing temperature, while the rest of the spectra remain unchanged (Fig. 2). The proportional increase of the scattering intensity at various energies (lower two curves in Fig. 3) and also independent higher resolution NSE results show that this is not due to the broadening of the $\omega=0$ quasielastic central line, but to the increasing amplitude of a component with invariant line shape. The so-called $\beta$ relaxation process predicted by MC theories corresponds to this observation with a lineshape $\omega^{\alpha} / \omega_{\epsilon}^{\alpha}$. Experimentally $\alpha$ is compatible with values around -0.8 in $\mathrm{kCN}$, with a large error of 0.2 , however. The sharp increase of the $50 \mu \mathrm{eV}$ spectral density in Fig. 3 above some $90^{\circ} \mathrm{C}$ can be attributed to the on-set of the broadening of the $\omega=0$ line (which is expected to have a complex lineshape) and it is also confirmed by the 


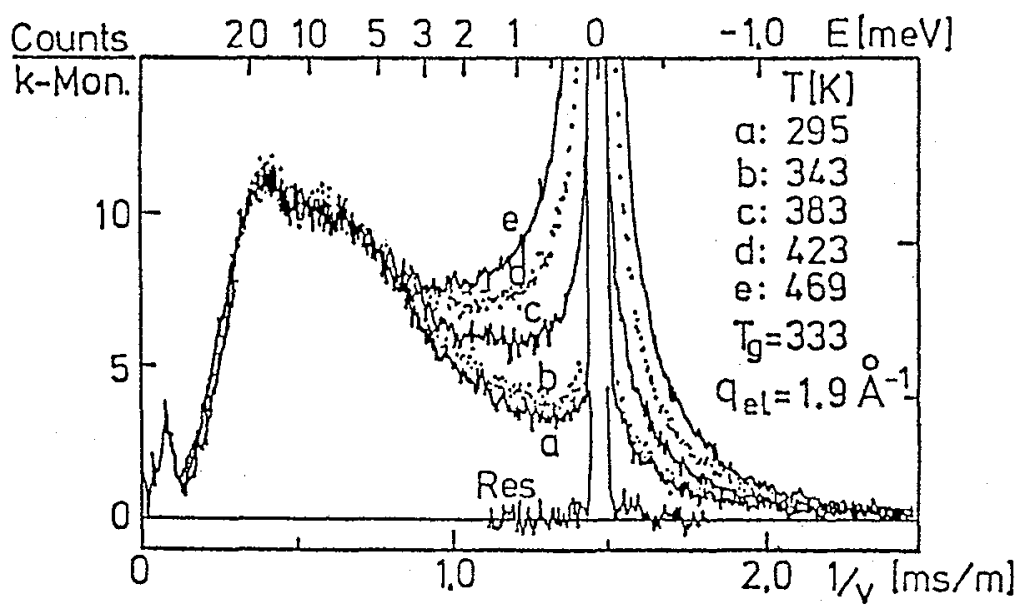

Fig. 2. Time-of-flight neutron scattering spectra in KCN at various temperatures ${ }^{2}$. The spectra are normalized by the Bose temperature factor in order to remove the trivial part of the temperature dependence characteristic of constant density of states. The relevant energy variable is indicated on the top scale.

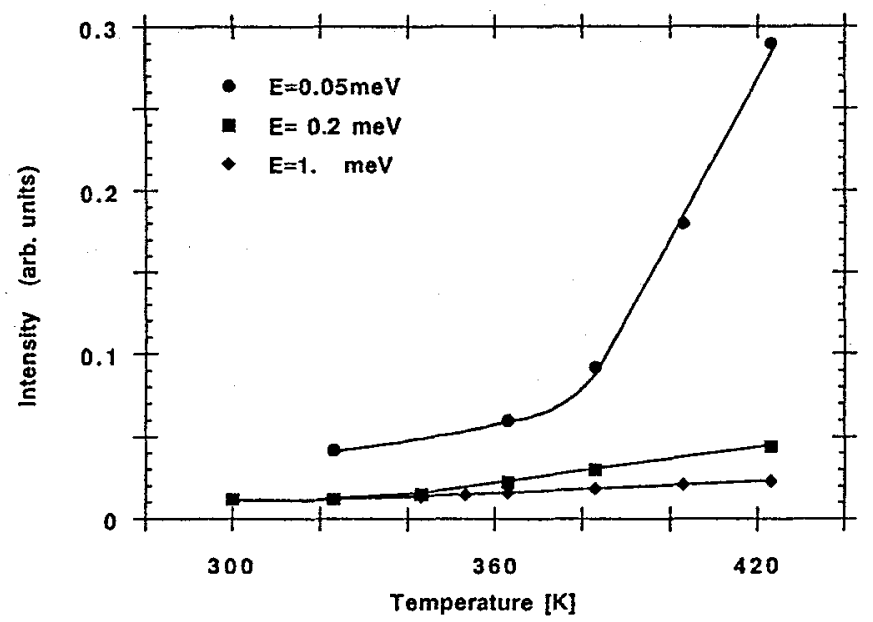

Fig. 3. Temperature dependence of the spectral intensities in Fig. 2. at two energies (lower curves) and at a smaller energy as observed by higher resolution backscattering spectroscopy. The upturn of the lower curves around $333 \mathrm{~K}$ is due to the onset of the " $\beta$ " relaxation, and that of the uppermost curve at about $365 \mathrm{~K}$ is due to the " $\alpha$ " relaxation. The lines are guides to the eyes. 
lack of similar upturn at higher energies, which in fact become affected by the line-broadening at higher temperatures only.

Another important feature of this anomalous scattering contribution, which we shall refer to as " $\beta$ " relaxation in what follows, is that its relative amplitude $h(q)$ in the structure factor $s(q) \simeq \int s(q, \omega) d \omega$ is rather independent of the wavenumber in a broad range (Fig. 4) as determined by the NSE method (which actually measures this energy integral within the mainly relevant energy range of $-1 \mathrm{meV}$ to $4 \mathrm{mev}$ ). More precisely $13, \mathrm{~h}(\mathrm{q})$ has a slight minimum at the maximum of the structure factor (also shown in Fig. 4) as predicted by MC. This relative $q$ independence of $\mathrm{h}(\mathrm{q})$ is of primary significance. Assumptions, that this process be due to some extra low energy excitations would lead to $h(q) \propto q^{2}$ or $q^{2} / s(q)$, respectively, depending on whether extended or localized modes are assumed. Due to the presence of several kinds of atoms in $\mathrm{KCN}$ the structure factor is rather substantial down to small $\mathrm{q}^{\prime} \mathrm{s}$ of $0.1-0.2 \mathrm{~A}^{-1}, \mathrm{i} . \mathrm{e}$. only an order of magnitude smaller than the peak in the atomic density structure factor $s(q)$ at $q_{0} \simeq 1.8 \AA^{-1}$ corresponding to the nearest atomic neighbours. A plateau ("prepeak") around $0.8 \AA^{-1}$ corresponds to the physically really characteristic length of the system, the cation-cation (anion-anion) distance, i.e. to the peak of the charge density correlation function. This fortunate situation allows us to observe $h(q)$ at rather small $q^{\prime} s$, where the MC predictions are expected to be valid best. Wavenumbers larger than $q_{0}$ should be considered with caution, because at these values scattering contributions from local atomic vibrations become gradually dominant, and these cannot be expected to follow the universal predictions of MC theories.

Thus, the " $\beta$ " relaxation process as observed here at $T \leqslant 90^{\circ} \mathrm{C}$ has the characteristics of a first, faster step of the structural relaxation, i.e. of the central $\omega=0$ quasielastic line. This observation is only explained by MC theories up to now.

\section{The " $\alpha$ " relaxation process}

The other remarkable unusual feature is the lineshape behaviour of the bulk (second step) of the structural relaxation at higher temperatures, the " $\alpha$ " process, as observed by NSE spectroscopy directly in the time domain at $q \sim q_{0}($ Fig. 5$)$.

The lines in the figure represent a common lineshape function of the Kohlrausch type

$$
\Phi(q, t)=f e^{-(t / \tau)^{\beta}}
$$

where $T$ was taken proportional to the measured bulk viscosity $T=B \eta(T)$ and the parameters $f, B$, and $\beta$ have been chosen to best fit the $T=176^{\circ} \mathrm{C}$ curve ( $f=0.9$ and $\beta=0.58$ ). This master function, which is very different from the usual exponential decay (Lorentzian spectral lineshape: $\beta=1$ ), quite well describes most of the data, with clear deviations at both the highest and lowest temperatures (the uppermost line is calculated for $112^{\circ}$, not for $93^{\circ} \mathrm{C} !$ ). No more permissive fitting would completely cure these "problems", however common fits to all temperatures give mathematically very small errors for $f$ and $\beta$ which are often heavily 


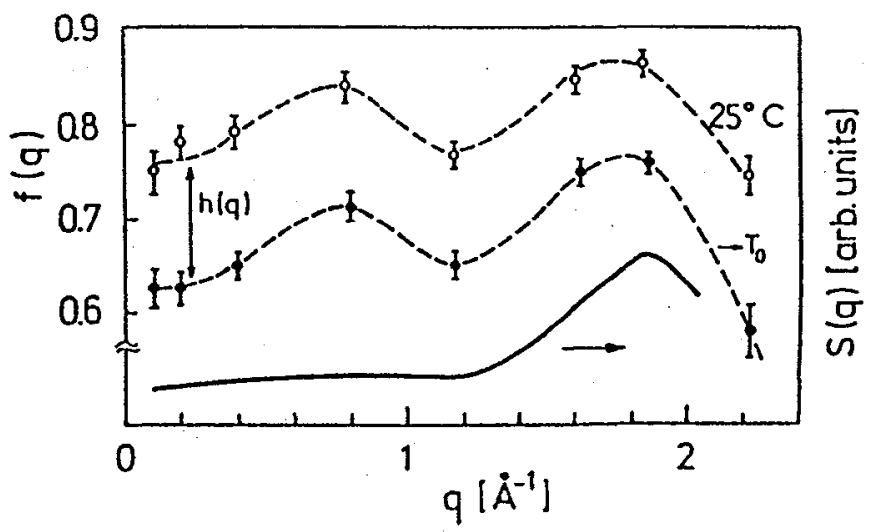

Fig. 4. Wavenumber dependence of the relative amplitudes $f(q)$ and $h(q)$ of the " $\alpha$ " and " $\beta$ " relaxation steps, respectively, in $\mathrm{KCN} . \mathrm{h}(q)$ is obtained as the difference between $f(q)$ at room temperature (where no " $\beta$ " relaxation component can be observed and the " $\alpha$ " relaxation is practically infinitely slow) and at the estimated critical temperature $T_{0}$, above which the two processes overlap significantly 13 .

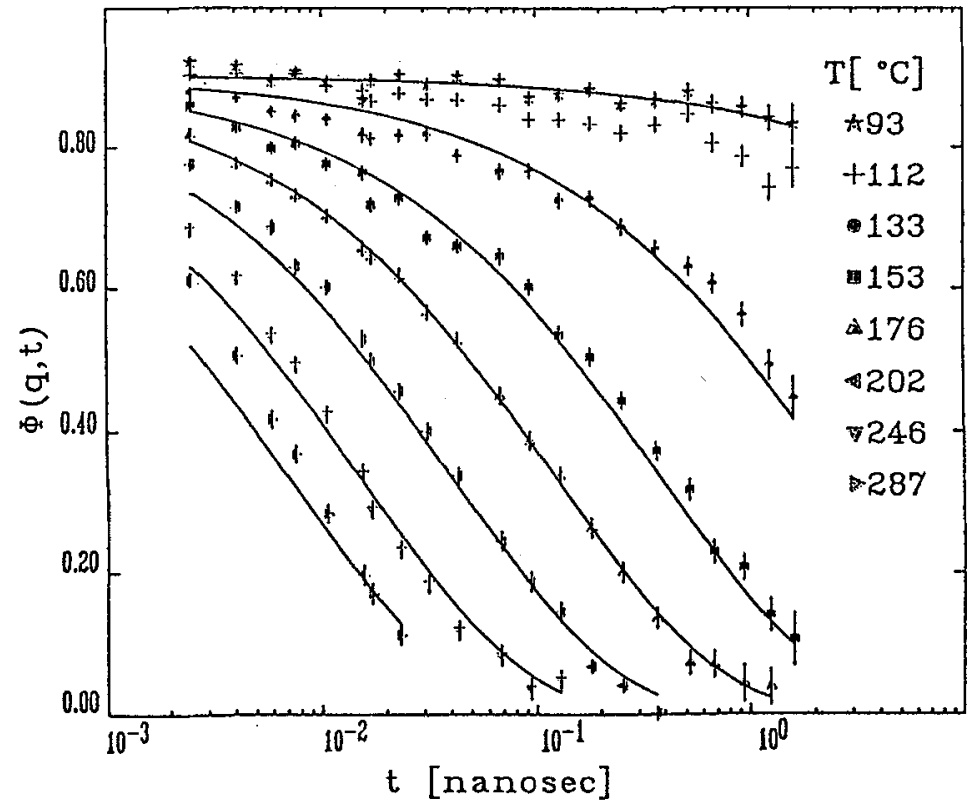

Fig. 5. Extended range NSE results on the temperature dependence of the time relaxation function (intermediate scattering function) at the peak of the structure factor $q_{0}$ in $\mathrm{KCN}$. The lines were obtained by shifting the time scale of a fit to the $176^{\circ} \mathrm{C}$ data proportionally with the change of bulk viscosity ${ }^{6}$. Note that the uppermost line corresponds to $112^{\circ} \mathrm{C}$. 
misinterpreted. A critical look at the errors on the lineshape parameters $f$ and $\beta$, which were obtained by individually fitting the data at different temperatures independently of other temperatures, is rather sobering: $3 \sigma$ errors (998 confidence level) run to some 0.05 and 0.12 , respectively, at best. By taking the best common value of $f \simeq 0.90$, it is clear that the other lineshape parameter $\beta$ becomes significantly temperature dependent (Fig. 6). One reason for this is the admixture of the " $\beta$ " relaxation process, in particular its tail towards longer times (tentatively identified with the so-called von schweindler relaxation). This is well signified by the faster drop of the $112^{\circ} \mathrm{C}$ data points in Fig. 5. than the calculated line. The non-zero slope of the $93^{\circ} \mathrm{C}$ data is entirely due to the " $\beta$ " relaxation process, assuming that $\tau$ continues to scale with viscosity $\eta$ and that the exponent $\beta$ remains larger than 0.3 , which is not quite obvious in Fig. 6 . Adding further parameters to the fit in order to include the " $\beta$ " contribution would blow up the errors, and the results of an analogous attempt ${ }^{6}$ to correct for this " $\alpha$ " and " $\beta$ " mixing is shown in the bottom part of Fig. 6 . A constant
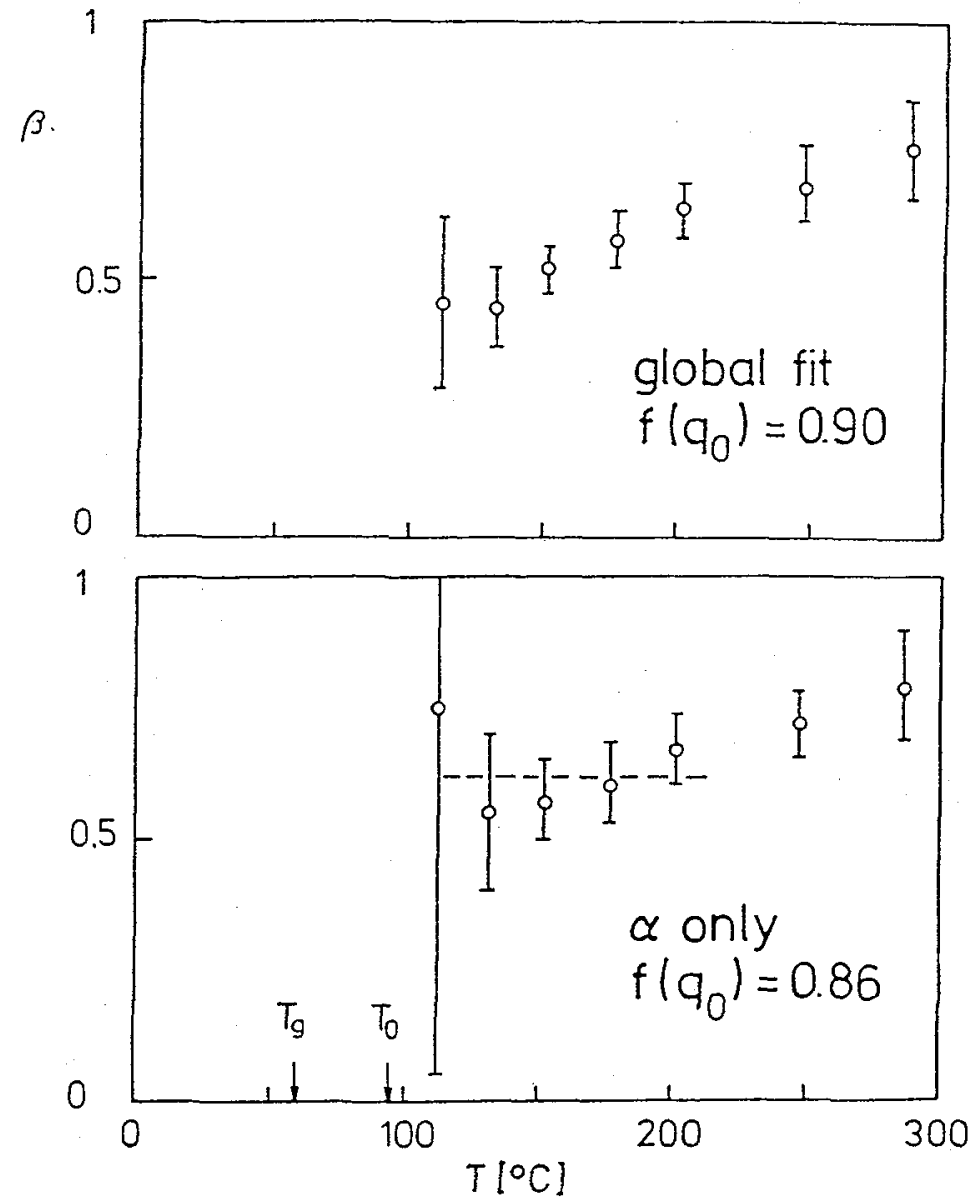

Fig. 6. Temperature dependence of the Kohlrausch lineshape exponent $\beta$ obtained by fitting the data in Fig. 5. at each temperature independently, but assuming a temperature independent amplitude $f$. Top: all data points considered; bottom: data points strongly influenced by the " $\beta$ " relaxation step disxegarded ${ }^{6}$. The error bars indicate $3 \sigma$ deviations, i.e. 99 \% confidence level. 
lineshape with $\beta \simeq 0.61$ now becomes possible over a temperature range of $100{ }^{\circ} \mathrm{C}$, however this hardly looks like a prove. Note, that the temperature dependence of the Kohlrausch exponent $\beta$ observed in macroscopic and light scattering measurements is much more rapid 14,15 , and this discrepancy between the two kinds of results remains, no matter what type of $f$ it is used.

MC theories predict an assymptotically constant lineshape of the " $\alpha$ " relaxation process (i.e. temperature independent $f$ and $\beta$ parameters in this case) upon approaching a critical temperature $T_{0}$, which is experimentally estimated to be at about $95{ }^{\circ} \mathrm{C}$ in this sample. It is seen, that the results are not incompatible with this assumption, however they do not lend much support to it either, except for the fact that the lineshape is strongly non-exponential. This latter very unusual prediction for a simple liquid without a network structure has also been suggested by other considerations 14 . Another MC theoretical expectation, the relative $q$-independence of the effective relaxation times is well born out, with a pronounced de Gennes type narrowing effect, however, around the wavenumber of the charge density correlation peak $\left(\sim 0.8 \AA^{-1}\right)$, cf. Fig. 7 , showing that this wavenumber indeed corresponds to the real characteristic dynamic length scale of the system. Usually this narrowing occurs at the peak of the structure factor instead.

\section{Concluding Remarks}

In quantitative respects MC theories predict a number of scaling relations

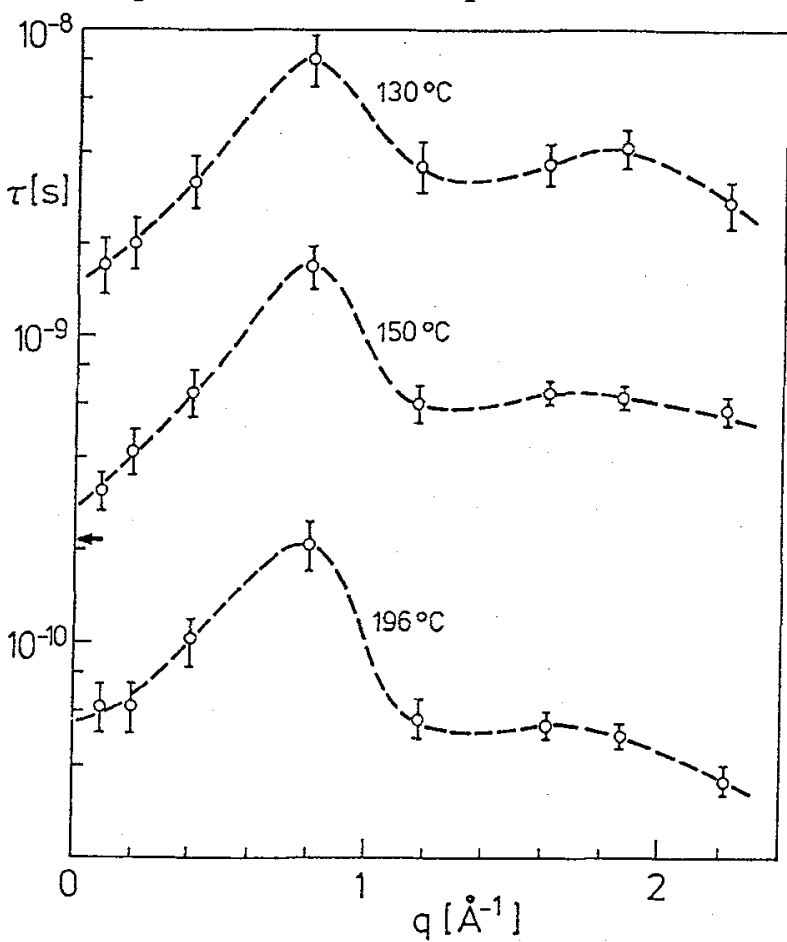

Fig. 7. Wavenumber dependence of the effective relaxation time $\tau$ of the " $\alpha$ " relaxation step in $\mathrm{KCN}^{1}$. The arrow indicates the value obtained at $150{ }^{\circ} \mathrm{C}$ and $q=0$ by Bxillouin light scattering ${ }^{15}$. The lines are mere guides to the eye. 
between exponents describing the " $\beta$ " relaxation lineshape, the temperature dependence of $\omega_{\varepsilon}$ and of $\eta$ or $\tau$. These relations can be forced to be fulfilled 16 by an appropriate choice of $T_{0}$. This, however, is again a compatibility check only, and provides little extra evidence for the theory. The problem is, that around $T_{0}$ thermally activated jump processes dominate the structural relaxation and no clear and reliable signature of $T_{0}$ remains. Actually, one should not try to fit data around $T_{0}$ by MC predictions, because just around this temperature this theory breaks down. Including the jump diffussion explicitely, on the other hand, would dramatically increase the number of free parameters and thus the ambiguity of interpretation. In sum we can conclude that MC theory - by now alone correctly predicts the strikingly new phenomenon of a two step structural relaxation dymamics in supercooled liquids just above the glass transition, and correctly describes the types of lineshape for both of these two processes at temperatures where they do not overlap in time. This is a strong qualitative evidence in favour of the theory. More quantitative comparisons between theory and experiment would only be meaningful, if total lineshape (" $\alpha$ " plus " $\beta$ ") calculations were available with a reduced number of free parameters. Since such type of results go beyond the universal features of the theory and become model dependent, realistic molecular dynamic calculations might be necessary.

\section{REFERENCES}

[1] F. Mezei, W. Knaak and B. Farago, Phys. Rev. Lett. 58, 363 (1987), and Phys. Scripta 19, 571 (1987).

[2] W. Knaak, F. Mezei and B. Farago, Europhys. Lett. I, 529 (1988).

[3] D. Richter, B. Frick and B. Farago, Phys. Rev. Lett. 61, 2465 (1988) and 64, 2921 (1990).

[4] F. Fujara and W. Petry, Europhys. Lett. 4, 921 (1987); W. Petry, M. Kiebel and H. Sillescu, in Dynamics of Disordered Materials, D. Richter et. al. eds. (Springer Verlag, Heidelberg, 1989), p. 58.

[5] W. Doster, S. Cusack and w. Petry, ibid. p. 120.

[6] F. Mezei, J. Non-Cryst. Solids, 131-133, 317 (1991).

[7] C.A. Angell, J. Phy. Chem. Solids, 49, 863 (1988).

[8] E. Leutheusser, Phys. Rev. A229, 2765 (1984); U. Bengtzelius, W. Götze and A. Sjölander, J. Phys. C. 17, 5915 (1984); S.P. Das, G.F. Mazenko, S. Ramaswamy and F.F. Toner, Phys. Rev. Lett. $\underline{54}, 118$ (1985).

[9] W. Götze, in Liquids, Freezing and the Glass Transition, J.P. Hansen, D. Levesque and J. Zinn-Justin, eds. (North Holland, Amsterdam, 1991), p. 287.

[10] W. Götze, E. Leutheusser, and S. Yip, Phys. Rev. $\underline{\text { A23 }, ~} 2634$ (1981) and A24, 1008 (1981).

[11] L. Sjögren, in Basic Features of the Glassy state, J. Colmenero and A. Alegria, eds. (World Scientific, Singapore, 1990), p. 297

[12] F. Mezei, in Liquids, Freezing and the Glass Transition, J.P. Hansen, D. Levesque and J. Zinn-Justin, eds. (North Holland, Amsterdam, 1991), p. 629.

[13] F. Mezei, in Dynamics of Disordered Materials, D. Richter et. al. eds. (Springer Verlag, Heidelberg, 1989), p. 164.

[14] K.L. Ngai, A.K. Rajagopal and C.Y. Huang, J. Appl. Phys. 55, 1714 (1984).

[15] M. Grimsditch and L.M. Torell, in Dynamics of Disordered Materials, D. Richter et. al. eds. (Springer Verlag, Heidelberg, 1989), p. 196.

[16] F. Mezei, in: Basic Features of the Glassy state, J. Colmenero and A. Alegria, eds. (World Scientific, singapore, 1990), p. 152 